La Revue

des Droits

de l'Homme

\section{La Revue des droits de l'homme}

Revue du Centre de recherches et d'études sur les droits fondamentaux

$20 \mid 2021$

Revue des droits de l'homme $-\mathrm{N}^{\circ} 20$

\title{
Physionomie générale du corpus QPC et méthodologie de la recherche
}

Thomas Acar, Véronique Champeil-Desplats, Antonin Gelblat et Stéphanie Hennette Vauchez

\section{(2) OpenEdition}

\section{Journals}

Édition électronique

URL : https://journals.openedition.org/revdh/12680

DOI : 10.4000/revdh. 12680

ISSN : 2264-119X

\section{Éditeur}

Centre de recherches et d'études sur les droits fondamentaux

\section{Référence électronique}

Thomas Acar, Véronique Champeil-Desplats, Antonin Gelblat et Stéphanie Hennette Vauchez, "Physionomie générale du corpus QPC et méthodologie de la recherche », La Revue des droits de I'homme [En ligne], 20 | 2021, mis en ligne le 01 juillet 2021, consulté le 07 juillet 2021. URL : http:// journals.openedition.org/revdh/12680; DOI : https://doi.org/10.4000/revdh.12680

Ce document a été généré automatiquement le 7 juillet 2021.

Tous droits réservés 


\title{
Physionomie générale du corpus QPC et méthodologie de la recherche
}

\author{
Thomas Acar, Véronique Champeil-Desplats, Antonin Gelblat et Stéphanie \\ Hennette Vauchez
}

12020 allait sans aucun doute constituer un moment favorable à diverses entreprises de bilan de la Question prioritaire de constitutionnalité. Comme toute réforme juridique d'ampleur, cette nouvelle procédure de contrôle de constitutionnalité, entrée en vigueur le $1^{\mathrm{er}}$ mars 2010 , d'emblée promise à un avenir capital, voire glorieux ${ }^{1}$, est en effet longtemps restée difficile à appréhender. Les chiffres bruts indiquaient assez vite que la QPC avait « rencontré son public » : à partir de la toute première décision rendue le 28 mai $2010^{2}$, ce ne sont pas moins de 64 décisions QPC qui ont été rendues sur l'année 2010 - chiffre à comparer à celui de 24 décisions DC. L'année suivante, le Conseil rendait 110 décisions $\mathrm{QPC}$ contre 23 décisions $\mathrm{DC}^{3}$. Unanimement, c'est un succès, parfois même qualifié de "fulgurant $»^{4}$, qui est salué.

Difficile pour autant, sans le recul et les outils d'analyse nécessaires, de dresser un bilan global avant que s'écoulent un certain nombre d'années. Certes, quelques mouvements ponctuels ou de fond ont progressivement été identifiés. La Cour de cassation a ainsi pu apparaître initialement réservée, voire hostile à la procédure - c'est en ce sens qu'ont $\mathrm{pu}$ être lues quelques décisions retentissantes par lesquelles elle donnait l'impression de ne pas vouloir laisser les sages de la rue Montpensier pénétrer son office ${ }^{5}$. De premiers bilans, à trois ans ${ }^{6}$ ou cinq ans ${ }^{7}$, commençaient de dessiner des tendances : redéfinition de l'office du juge ordinaire, enclenchement d'importantes évolutions du droit positif... Mais l'écoulement d'une décennie complète fournit l'occasion d'une remise à plat plus globale, afin que la procédure puisse être confrontée aux objectifs qui lui étaient assignés $a b$ initio. La protection constitutionnelle des droits et libertés s'estelle trouvée renforcée, enrichie, par la QPC ? De nouveaux droits ont-ils été consacrés ? De nouvelles méthodes de contrôle ont-elles été mises en place? La figure de la QPC, «sentinelle démocratique $»^{8}$ de l'Etat de droit, est-elle confortée ? A-t-on réellement vu 
« l'individu» pénétrer le cœur de « l'espace constitutionnel $»^{9}$ de la défense des droits et libertés?

3 Il existe, assurément, de nombreuses manières de dresser le bilan de dix années de pratique effective d'une procédure telle que la QPC. Le Conseil constitutionnel luimême a d'ailleurs souhaité y procéder, publiant à cette occasion de nouvelles séries de données statistiques ${ }^{10}$, et confiant à Mme Nicole Maestracci, l'une de ses membres, la responsabilité d'un appel à projets de recherche à destination du monde académique en vue de l'organisation d'une manifestation publique ${ }^{11}$. Dans ce cadre comme dans d'autres, un foisonnement de données a donc été produit: des études sectorielles (la QPC et le droit de la culture, la QPC et le droit de l'environnement, la QPC et le droit fiscal...), thématiques (les porteurs de la QPC, l'effet utile de la QPC...), ou techniques (le traitement de la QPC par les juridictions du fond, Procédure législative et QPC...).

La présente étude entend contribuer à ce moment de recul et d'évaluation de la QPC, en posant un certain nombre de questions transversales à l'ensemble du corpus de décisions rendues depuis 2010 par le Conseil constitutionnel dans le cadre de cette procédure. Elle entend, en d'autres termes, interroger la QPC au prisme de sa fonction de définition d'un nouveau niveau de protection constitutionnelle des droits et libertés, tant au regard de la manière dont elle a été promise par ses concepteurs qu'au regard des modalités concrètes de sa mise en œuvre au cours de la décennie écoulée. Un groupe de chercheurs s'est donc réuni au sein du CREDOF de l'Université Paris Nanterre, commençant de travailler à la définition d'une méthodologie de recherche reposant sur plusieurs piliers. Il a d'abord été décidé de procéder à un travail de codage de l'ensemble des décisions rendues par le Conseil constitutionnel dans le cadre de la QPC : du 28 mai 2010, date de la première décision, au 31 décembre 2019 (soit, près de dix années de contentieux), ce sont donc 730 décisions qui ont été analysées. Elles l'ont été au regard des clés d'analyse suivantes :

- Données propres à la décision : numéro, année et juridiction de renvoi

- Données propres au requérant: qualité (personne physique ou morale), raison sociale (entreprise, collectivité locale, association...)

- Données propres à la représentation du requérant (voire, des tiers intervenants) : avocat individuel, avocat au conseil...

- Données propres aux domaines dont relèvent les dispositions législatives attaquées : droit de l'environnement, droit des personnes, droit fiscal, droit pénal...

- Données propres aux moyens soulevés : substantiels ou procéduraux

- Données propres au type de contrôle exercé: proportionnalité, erreur manifeste d'appréciation, type de conciliation opéré...

- Données propres à l'issue du contrôle : conformité ou non-conformité (partielle ou totale), réserves, modulation dans le temps...

- Données propres à l'institution de contrôle: nombre de membres présents, déports éventuels...

5 Toutes ces clés d'analyse ont nécessité un long travail méthodologique de définition, d'ajustement, d'affinement et de classement qui est détaillé ci-bas (II). Elles ont permis la production d'une base de données qui permet de saisir, et donc de décrire, la physionomie générale du corpus juridique généré par dix années de QPC (I). 


\section{Physionomie générale du corpus QPC}

6 Ce sont donc 730 décisions qui ont été rendues par le Conseil constitutionnel entre 2010 et $2019^{12}$. Ce chiffre permet à lui seul de mesurer l'importance quantitative de la QPC dans l'activité du Conseil constitutionnel, qui contraste avec le fait qu'au 31 décembre 2019, le Conseil n'avait rendu « que " 796 décisions DC - alors même que cette procédure-là de contrôle existe depuis 1958 (v. tableau $\left.{ }^{13}\right)$.

\begin{tabular}{|l|l|l|l|l|l|}
\hline \multicolumn{2}{|l|}{ Contrôle de constitutionnalité } & Elections & Autres (divers) & Total \\
\hline DC (art. 61 C) & QPC (art. 61-1 C) & Sous-total & & & \\
\hline 795 & 730 & $1525^{14}$ & 3827 & 520 & 5878 \\
\hline
\end{tabular}

7 Ainsi, en dix années seulement, la QPC en est venue à représenter $47,8 \%$ de la part d'activité du Conseil constitutionnel consacré au contrôle de la constitutionnalité des lois. Il s'agit donc d'un engouement considérable des justiciables pour cette nouvelle voie de droit. Certes, diverses critiques relatives au manque d'effet utile de la QPC pourraient bien, dans le futur, relativiser cet engouement. C'est en effet dès la décision DC qu'il avait rendue sur la loi organique relative à l'application de l'article 61-1 de la Constitution que le Conseil constitutionnel avait consacré l'exigence d'effet utile de la QPC pour le requérant ${ }^{15}$. Pour autant, et singulièrement dans les années récentes, celleci semble avoir parfois cédé le pas devant d'autres exigences concurrentes. Si le considérant de principe demeure ${ }^{16}$, nombre des déclarations d'inconstitutionnalité prononcées dans le cadre de la QPC ont été cantonnées, du fait de la manière dont le Conseil a choisi de recourir au pouvoir de modulation dans le temps des effets de ses décisions en vertu de l'article 62 de la Constitution, à des effets platoniques. Une récente étude établit ainsi que ce ne sont pas moins de $32 \%$ des décisions rendues en QPC qui, en dépit du principe de l'effet utile qui demeure affirmé, en sont totalement dépourvues du point de vue du requérant ${ }^{17}$; certains contentieux spécifiques, comme celui relatif à la loi du 3 avril 1955 relative à l'état d'urgence qui demeura en vigueur pendant près de deux années à la suite des attentats du Bataclan et du stade de France, semblent particulièrement emblématiques de cette neutralisation de l'effet utile de la $\mathrm{QPC}^{18}$. Cette question représente, dès lors, l'une des questions qu'aura permis de mettre au jour la grande entreprise de bilan de dix années de QPC; et elle compte certainement parmi celles qui seraient, le cas échéant, le plus susceptibles de " coûter " au Conseil constitutionnel en termes de succès de la procédure et d'engouement à son endroit.

8 La répartition par années de la QPC est relativement stable, autour de 65 décisions par an, exception faite des années 2011 et 2016 où les chiffres sont montés, respectivement, à 114 et 81 décisions. Le pic relatif de l'année 2011 est généralement expliqué par la nouveauté d'une procédure qui était annoncée et attendue, de sorte qu'il existait, au moment de l'entrée en vigueur de la QPC, un "stock» d'affaires en attente. Quant à celui de 2016, il peut être en partie expliqué par la situation exceptionnelle qu'a représenté l'état d'urgence déclenché à la suite des attentats de novembre 2015 qui, ayant duré près de deux années, a suscité pas moins de 9 QPC à lui seul ${ }^{19}$. 
9 Comme on le sait, c'est soit par la Cour de cassation soit par le Conseil d'État que les QPC peuvent être transmises au Conseil constitutionnel. De ce point de vue, l'observation d'une décennie de QPC n'altère pas l'analyse qui pouvait déjà être faite par Christine Maugüé sur la base de cinq années d'observations ${ }^{20}$ : les deux ordres juridictionnels renvoient à parts équivalentes, sinon égales ${ }^{21}$. Le Conseil d'État est ainsi à l'origine de 375 décisions (51,4 \% du total), et ce chiffre s'élève à 354 (48,5\%) pour la Cour de cassation ${ }^{22}$. Le temps des polémiques fortes qui, dans les premiers mois de l'entrée en vigueur de la QPC, avaient été suscitées par ce qui était perçu comme une volonté de la Cour de cassation de tenir la nouvelle procédure en échec, semble donc révolu ${ }^{23}$.

10 Plus intéressantes sont les données consolidées relatives au profil des requérant-es, qui révèlent qu'il ne s'agit de personnes physiques que dans 49,5\% des QPC (soit 361 décisions). On note également qu'il est très rare que ces personnes physiques requérantes soient des femmes: elles ne soulèvent des QPC que dans 9,7\% des cas, tandis que 37,9\% d'entre eux sont initiés par des hommes (dans 10,8 \% des cas, hommes et femmes portent ensemble une QPC). Cette part relativement modeste des personnes physiques parmi les requérant-es de la QPC doit donc être lue parallèlement au fait que dans $41,5 \%$ des décisions (soit un chiffre de 303 décisions), le ou les requérants sont des personnes morales; et il s'agit à la fois de personnes physiques et de personnes morales dans $9 \%$ des cas. Ce chiffre met sur la piste d'un premier élément d'analyse : les « droits et libertés que la Constitution garantit ${ }^{24}$ dont la protection est recherchée au moyen de la QPC ne sont pas exclusivement, loin de là, ceux des personnes physiques - de l'individu censé "s'approprier les principes constitutionnels " grâce à cette procédure ${ }^{25}$. Un tel constat est congruent, assurément, avec un autre, opéré de longue date, relativement au fait que les personnes morales sont, tout comme les personnes physiques, titulaires de droits dits fondamentaux. On sait que cette question a, ici comme ailleurs, suscité d'importants interrogations et travaux ${ }^{26}$; il est intéressant de l'affronter à nouveaux frais à la lumière de la QPC. A fortiori dès lors que l'analyse plus fine permet de souligner que, parmi cette importante proportion de décisions suscitées par des personnes morales, ce sont les porteurs d'intérêts économiques qui sont majoritaires. On dénombre ainsi 202 décisions rendues à l'initiative de QPC posées par des entreprises ou fédérations d'entreprises, ce qui représente les deux tiers des questions posées par les personnes morales et plus d'un quart de l'ensemble des décisions QPC (27,7\%). A titre de comparaison, les personnes morales de droit public sont à l'origine de 60 décisions, les associations dont l'objet social n'est pas la défense des droits humains sont à l'origine de 49 décisions et les syndicats, de 44 d'entre elles. De manière notable, les associations et ONG dont la raison sociale est directement en lien avec la problématique des droits humains (à l'instar de la Ligue des droits de l'Homme, de l'observatoire international des prisons, etc.) ne sont à l'origine que de 47 décisions (soit 6,4\% de l'ensemble des décisions QPC). Certes, elles peuvent intervenir autrement que comme requérantes : elles peuvent être tierces intervenantes ou encore, participer et contribuer activement à des interventions produites par d'autres. Reste que la pleine participation comme requérantes des associations de défense des droits humains demeure relativement faible, ce qui vient renforcer le constat précédent: quelle que soit l'importance aujourd'hui acquise par cette procédure de contrôle de constitutionnalité, elle n'est pas exclusivement ni même majoritairement investie par les organisations dont l'objet social est la défense directe des droits fondamentaux des personnes. Ceci ne signifie pas, certes, qu'un tel objectif n'est pas poursuivi par une 
proportion importante de personnes physiques; et de fait, on sait que certaines décisions suscitées par des requérants individuels soulèvent directement des questions de droits humains; elles peuvent même être portées (défendues) grâce à des avocats dont on connaît, par ailleurs, les liens avec des organisations de défense des droits humains ${ }^{27}$. Il n'en reste pas moins intéressant de constater que, tandis que les entreprises et fédérations d'entreprises représentent $27,7 \%$ de l'ensemble des décisions QPC rendues, les associations de défense des droits humains ne sont à l'origine que de $6.4 \%$ d'entre elles

11 Les conditions de représentation des requérants indiquent, quant à elles, d'autres traits intéressants. De manière globale, les données que nous avons produites établissent que les requérants étaient représentés dans $95 \%$ des cas et le plus souvent par des avocats au Conseil d'Etat et à la Cour de cassation. Deux éléments ici méritent plus particulièrement d'être soulignés. Alors même que la procédure de QPC ne requiert pas que la représentation du requérant soit assurée par un avocat, les chiffres révèlent d'une part que les requérants sont non seulement le plus souvent représentés. D'autre part, on observe que, parmi ceux-ci, il existe un petit groupe de structures semblant avoir consenti un investissement notable, en forme de spécialisation, dans le contentieux de la QPC. Le premier élément peut s'expliquer relativement aisément par un effet de sentier de dépendance juridictionnel : la représentation peut être nécessaire à une étape spécifique du parcours qu'est le filtrage opéré par les cours suprêmes. La QPC s'inscrit en effet généralement dans un cheminement juridictionnel long et relativement complexe, au sein duquel ne peut être ignorée l'importance décisive de cette étape spécifique du filtre ${ }^{28}$. Ces éléments sont de nature à opérer comme une incitation forte au fait de confier son dossier, d'emblée, à un avocat voire, à un avocat aux Conseils. Quant au second élément, il ressort des données constituées dans le cadre de la présente recherche. Il nous est en effet apparu qu'un certain nombre de grosses structures juridiques d'avocats aux Conseils faisaient figure dans le contentieux de la QPC de repeat players. Afin de saisir, mesurer et documenter cette réalité, nous avons donc procédé à un codage spécifique des modalités de représentation des requérants en QPC. Après avoir listé l'ensemble des avocat-es représentant les requérants, nous les avons classés en fonction du nombre de fois où ils étaient intervenu-es en QPC (soit comme représentants du requérant, soit comme représentants d'un autre intervenant). Les résultats de cette opération furent les suivants :

\begin{tabular}{|l|l|l|l|l|}
\hline 1 ou 2 affaires & De 3 à 17 affaires & De 25 à 33 affaires & Plus de 50 affaires & 99 affaires \\
\hline 283 avocats & 62 avocats & 2 avocats & 2 avocats & 1 avocat \\
\hline
\end{tabular}

12 La première colonne représente donc la catégorie des avocats sans spécialisation en $\mathrm{QPC}$, et qui ne se sont donc trouvés liés à cette procédure que par le hasard d'une affaire leur ayant été confiée. La seconde catégorie a été distinguée au motif qu'il a été considéré qu'à partir de 3 affaires (et a fortiori au-delà), l'hypothèse d'une petite reconnaissance de la capacité et de l'expérience de l'avocat ou du cabinet en la matière pouvait être formulée. Quant aux trois catégories restantes, par ordre croissant, elles désignent quelque chose comme une captation du contentieux QPC par un petit nombre de structures - la SCP Spinosi et Sureau arrivant largement en tête, avec 99 QPC prises en charge. Ces chiffres suffisent à poser un élément d'analyse intéressant: en une 
décennie, la QPC a donc mené à une évolution notable de la profession d'avocat qui, non seulement, a consenti un investissement authentique dans le droit constitutionnel - une branche du droit qu'elle avait jusque-là largement négligée et qui a donc été investie de manière proactive par un petit segment de la profession qui est parvenu à s'imposer comme central sinon incontournable pour la QPC dans son ensemble. L'avocat, en ce sens, est bel et bien aujourd'hui un acteur majeur du procès constitutionnel ; il s'est largement « approprié » la procédure ${ }^{29}$.

13 Au-delà de ces éléments relatifs à l'origine des QPC (requérants, représentants...), l'étude des sujets et domaines dans lesquels la QPC est mobilisée est riche d'enseignements. L'étude menée par exemple en 2015 par Christine Maugüé avait pu relever que certains domaines spécifiques avaient plus particulièrement suscité des censures QPC ; elle listait notamment le droit pénal, le droit de l'environnement ou le droit fiscal ${ }^{30}$. Mais elle n'avait pas cherché à identifier quels étaient les domaines du droit qui, indépendamment de l'issue du litige, avaient suscité des QPC. Nos données révèlent que le droit fiscal est bien le domaine suscitant le plus grand nombre de QPC ( $\mathrm{n}=162$, soit $22,2 \%$ du corpus), suivi par le droit de la procédure pénale ( $\mathrm{n}=103$, soit $14,1 \%)$ et le droit des entreprises $(n=89$, soit $12,2 \%)$. D'autres domaines, tels que le droit de l'environnement $(n=30$, soit $4,1 \%)$, le droit des étrangers $(n=28$, soit $3,8 \%)$ ou le droit pénitentiaire $(n=9$, soit $1,2 \%)$ demeurent bien plus marginaux. A propos de ce classement, il faut toutefois préciser l'importance qui s'attache à la définition des catégories retenues. Ainsi par exemple, l'étude de Christine Maugüé souligne l'importance du droit pénal dans le contentieux de la QPC; mais il nous est apparu nécessaire d'affiner cette vaste catégorie, afin notamment que soient distinguées les affaires concernant la procédure pénale, le droit pénal substantiel ou encore le droit pénal des affaires qui, par définition, concerne l'activité économique. La sélection des catégories pertinentes pour rendre compte des grands domaines dont relèvent les dispositions législatives attaquées au fil de dix années de QPC est donc un travail de longue haleine pour qui veut rendre compte de la physionomie générale du corpus, qui est le produit à la fois inductif et déductif de nombreux allers-retours entre l'activité d'analyse et l'activité de codage.

14 Le résultat auquel nous avons abouti est le suivant. Au terme de la classification que nous avons retenue ${ }^{31}$, il apparait que c'est largement dans le domaine fiscal que la QPC est mobilisée : dans $22,2 \%$ des décisions, ce sont des dispositions législatives relatives à la matière fiscale qui sont attaquées, ce qui place ce premier domaine largement en tête. C'est la procédure pénale qui figure en seconde place, avec un taux cependant nettement inférieur de $14,4 \%$ des décisions, suivi de près par la catégorie «droit de l'entreprise et droit des affaires » $(12,2 \%)$ et par celle du droit administratif général $(11,6 \%)$. Le droit de l'environnement est loin derrière $(4,1 \%)$ avec le droit des étrangers $(3.8 \%)$, le droit du travail se positionnant lui à la $5^{\text {ème }}$ place $(9 \%)$ et le droit pénitentiaire à la toute dernière $(1,2 \%)$.

\begin{tabular}{|l|l|l|}
\hline & Fréquence & Pourcentage \\
\hline Droit fiscal & 162 & 22.2 \\
\hline Droit de la procédure pénale & 103 & 14.1 \\
\hline
\end{tabular}




\begin{tabular}{|l|l|l|}
\hline Droit des entreprises / Droit des affaires & 89 & 12.2 \\
\hline Droit administratif général & 85 & 11.6 \\
\hline Droit du travail & 66 & 9 \\
\hline Droit pénal & 63 & 8.6 \\
\hline Droit des collectivités locales & 58 & 7.9 \\
\hline Droit social (Sécurité sociale et protection sociale) & 55 & 7.5 \\
\hline Droit des personnes (état civil, famille, successions...) & 36 & 4.9 \\
\hline Droit disciplinaire & 34 & 4.7 \\
\hline Droit de l'environnement & 30 & 4.1 \\
\hline Droit pénal des affaires & 29 & 4 \\
\hline Droit des étrangers & 28 & 3.8 \\
\hline Droit de la communication & 27 & 3.7 \\
\hline Outremer & 60 & 2.3 \\
\hline Droit pénitentiaire & 37 & 1.2 \\
\hline Autres & 3.2 \\
\hline & 36 & \\
\hline
\end{tabular}

15 C'est notamment lorsqu'elles sont couplées à l'analyse des moyens soulevés que ces données sont intéressantes. Dans $88,1 \%$ des cas, au moins un moyen substantiel est soulevé à l'appui d'une QPC. Parmi eux, c'est le principe d'égalité qui arrive largement en tête : il est invoqué dans $65,1 \%$ des décisions, sous ses différentes facettes : égalité devant la loi (art. 6 de la DDHC, 38,4\%), égalité devant les charges publiques (art. 13 de la DDHC, $19 \%)$, principe d'égalité non spécifié (7,7\%). Ces chiffres indiquent à la fois la plastique du principe constitutionnel d'égalité ${ }^{32}$ et, corrélativement, l'intérêt qu'il peut représenter du point de vue d'une multitude de requérants défendant différents types d'intérêts. On le voit ainsi mobilisé pour plaider l'inconstitutionnalité de la loi pénale tout autant que de la loi fiscale ou pour souligner les effets produits par l'application de tels principe ou procédure. Le deuxième moyen substantiel le plus fréquemment invoqué à l'appui des QPC est celui de la violation de la liberté individuelle; nous avons regroupé sous cette appellation l'ensemble des requêtes arguant de la méconnaissance soit de l'article 66 de la Constitution, soit de celle des articles 2 et 4 de la Déclaration des droits de l'Homme et du Citoyen, soit encore de celle d'autres droits lorsqu'ils étaient invoqués dans un sens qui les rattachait à des préoccupations de liberté individuelle, à l'instar, par exemple, du droit de propriété. Égalité et Liberté se situent donc au sommet des moyens substantiels invoqués en QPC. Ils sont suivis, à hauteur de $19,9 \%$ des décisions, par les articles 7, 8 et 9 de la DDHC, qui forment le cœur des garanties pénales modernes: légalité, nécessité et proportionnalité des peines, 
présomption d'innocence. Ici encore, ce résultat est congruent avec le fait que le droit pénal et le droit de la procédure pénale figurent en bonne place des domaines d'appartenance des dispositions législatives attaquées par voie de QPC. Un peu plus bas, ce sont les libertés économiques dont les requérant-es se plaignent qu'elles sont méconnues par la loi. Nous avons classé comme relevant de cette catégorie la liberté d'entreprendre, la liberté du commerce et de l'industrie, la liberté contractuelle ou encore le droit de propriété. Le fait que cette catégorie de moyens soit invoquée dans $15,6 \%$ des affaires traitées en QPC est intéressant. Il mérite d'être mis en parallèle avec l'observation précédente relative aux requérants de la QPC, et à la forte représentation parmi eux des personnes morales porteuses d'intérêts économiques, et confirme des analyses déjà menées ${ }^{33}$ sur l'importance de la QPC pour les libertés économiques. Il mérite également un approfondissement de l'étude de la part des libertés économiques dans le contentieux de la QPC. Les libertés collectives occupent quant à elles une place bien modeste, puisqu'elles ne sont invoquées que dans 37 décisions seulement - soit une fois de moins que le principe de libre administration des collectivités territoriales. A la dernière place, la Charte de l'environnement n'apparaît que dans $2,5 \%$ des cas, ce qui confirme la faible part du droit de l'environnement dans le contentieux de la QPC.

16 Quant aux moyens procéduraux invoqués à l'appui des QPC dans $51 \%$ des cas, ils relèvent le plus souvent de considérations liées soit au fonctionnement de la justice (droit au recours effectif, autorités des décisions du Conseil constitutionnel, droits de la défense..., 32,3\% des cas) soit à la sécurité juridique (non-rétroactivité..., 18,4\%). Plus occasionnellement, ce sont des principes relatifs à la procédure parlementaire à la légistique dont la violation est alléguée, qu'il s'agisse de l'incompétence négative du législateur $(16,4 \%)$ ou de la qualité de la loi $(4,8 \%)$.

17 Puisque la QPC représente une nouvelle voie d'exercice de contrôle de la constitutionnalité des lois, il est également paru intéressant de s'intéresser aux modalités du contrôle déployé par le Conseil constitutionnel. On peut en effet s'interroger d'une part sur la question de savoir si la QPC a, comme le contrôle fondé sur l'article 61 avant lui, généré des techniques de contrôle nouvelles ou spécifiques; on peut également chercher à mesurer si le contrôle en QPC, parce qu'il porte spécifiquement sur "les droits et libertés que la Constitution garantit», s'apparente plus systématiquement à un contrôle de la proportionnalité dont la doctrine répète à l'envi qu'elle constitue le parangon du contrôle juridictionnel en la matière. Ici encore, la physionomie du contentieux de la QPC telle qu'elle ressort de la base de données que nous avons constituée est, dès l'étape de la description, intéressante. Saute ainsi d'emblée aux yeux la très faible proportion de décisions dans lesquelles le triple test de proportionnalité est explicitement exercé : on ne le trouve que dans 3,4\% des décisions du corpus global ${ }^{34}$. A l'inverse, et par contraste, on dénombre plus de $10 \%$ des décisions qu'il est possible d'apparenter à un contrôle de l'erreur manifeste du fait de la présence, dans la décision, d'expressions de type «erreur manifeste », ou "atteinte (non) manifestement» disproportionnée ou déséquilibrée. De la même manière, du point de vue de la structure du raisonnement, on est encore frappé par le fait que le schéma classique de la conciliation entre droits et libertés constitutionnels qui avait largement marqué, au moins jusque dans les années 1990, le contrôle a priori de la constitutionnalité de la loi semble avoir laissé largement place à un contentieux de la pondération entre d'une part, droits et libertés constitutionnels et d'autre part, intérêts collectifs de divers ordres. Si l'on prend ainsi la décision IVG de $2001^{35}$ comme 
parangon de ce modèle de la conciliation, on ne peut que constater que le schéma qui voyait s'affronter le principe de sauvegarde de la dignité humaine, d'un côté, et la «liberté de la femme » qui découle de l'article 2 de la Déclaration de 1789 de l'autre, ne semble plus tellement correspondre au raisonnement du Conseil constitutionnel tel qu'il se donne à voir au prisme de la QPC.

Les opérations de confrontation entre deux droits et libertés constitutionnels (ou davantage) sont en effet rares; elles ne représentent qu'1,8\% du corpus analysé. En revanche, pour plus de la majorité des décisions, on voit le Conseil constitutionnel mettre en balance une liberté ou un droit constitutionnellement garanti avec un intérêt collectif : l'intérêt général est invoqué dans $24,9 \%$ des cas, l'ordre public dans $9 \%$ des cas, la lutte contre la fraude (notamment en matière fiscale) dans $8,9 \%$ des cas et les autres intérêts collectifs dans $10,7 \%$ des cas, l'ensemble de ces références concernant un total de 387 décisions. En outre, la technique dite de l'effet «cliquet» ou «artichaut », popularisée par le doyen Favoreu dans les années 1990 et selon laquelle il convient que le législateur «ne prive pas de garanties légales » un droit ou une liberté que la constitution garantit, n'est mentionnée que dans $10 \%$ des QPC.

19 Enfin, nous nous sommes intéressés à l'issue des litiges soulevés par voie de QPC, afin de prendre la mesure de l'efficacité de cette procédure du point de vue de la contestation de la constitutionnalité des dispositions législatives attaquées. On sait l'efficacité potentielle de la QPC et l'intérêt qu'elle peut représenter pour le justiciable : pour celui qui souhaite obtenir une modification de la législation qui lui est applicable, la QPC présente en effet de nombreux attraits. Contrairement à l'alternative que représente, par exemple, le lobbying législatif, la procédure de QPC peut permettre d'atteindre des résultats comparables tout en demeurant relativement simple, relativement rapide (quelques mois) et potentiellement très efficace (ce n'est rien moins que l'abrogation de certaines dispositions qui peut être obtenue). Mais aboutitelle? Comme dans tout contentieux, les chiffres bruts de l'issue donnée aux requêtes sont difficiles à interpréter. Nos données indiquent que 66,6\% $(\mathrm{n}=486)$ des décisions étudiées ont été des décisions de conformité, 9,5\% des décisions de non-conformité partielle $(n=69)$ et $21,2 \%$ des décisions de non-conformité totale $(n=155) .14,4 \%$ $(\mathrm{n}=105)$ de l'ensemble de ces décisions étaient assortis de réserves - cette technique étant à peu près également représentée parmi les décisions de conformité (dont 18,3\% sont rendues sous réserve) et les décisions de non-conformité partielle (dont 18,8\% sont rendues sous réserve). Quelle que soit la difficulté de l'interprétation de tels chiffres, il paraît raisonnable de considérer qu'un taux de succès de la QPC de l'ordre de 30,7 \% (en partant de l'hypothèse que même une censure partielle est une victoire pour le requérant) est loin d'être négligeable - notamment, là encore, lorsqu'il est rapporté non seulement à celui dont on peut créditer les alternatives possibles (lobbying) mais encore à la rapidité et au coût modéré de l'action. Reste que, parmi les critiques faites à la procédure par ceux-là même qui la pratiquent (les requérants et leurs représentants), il faut compter avec l'aléa qui la caractérise - du fait, notamment, du pouvoir que détient le Conseil constitutionnel de moduler dans le temps les effets d'une éventuelle décision de non-conformités ${ }^{36}$. Si le Conseil adopte la position au terme de laquelle le requérant doit en principe bénéficier de la QPC qu'il a initiée ${ }^{37}$, son pouvoir de modulation vient souvent l'entraver. Or celui-ci est très largement utilisé par le Conseil, ici dans $12,1 \%$ des décisions QPC - ce qui nous a paru justifier une étude spécifiquement consacrée à cette question. 


\section{Méthodologie de la recherche.}

\section{Répondre ou ne pas répondre à un appel à projet financé par l'institution sur laquelle il porte?}

L'initiative du Conseil constitutionnel d'inviter par appel à projet des équipes de recherche à dresser un bilan des dix premières années écoulées de la procédure de QPC (1.1) est source de questionnements d'ordre tant méthodologique qu'épistémologique (1.2). Ces derniers ont conduit les membres de l'UMR 7074 à hésiter puis à renoncer à répondre l'appel à projet (1.3).

\subsection{Initiative}

21 Pour marquer les dix ans de la QPC, le Conseil constitutionnel a initié un appel à projet. L'objectif annoncé était de dresser le bilan de cette procédure, d'en identifier les succès mais aussi les limites. D'après les termes de l'appel, son ambition était plus précisément d'identifier «les aspects sociologiques de la QPC» et de réaliser des «bilans jurisprudentiels thématiques dans les principaux domaines juridiques concernés par la $\mathrm{QPC} »^{38}$. Ce faisant, le Conseil constitutionnel s'inscrivait dans une démarche volontariste de développement de la somme de connaissances disponibles sur la QPC afin d'éclairer les différents acteurs directement ou indirectement concernés (justiciables, avocats, institutions, chercheurs, etc..) ${ }^{39}$.

Le Conseil poursuivait ainsi un effort significatif consenti depuis quelques années pour opérer un retour réflexif sur sa propre activité. Cet effort se traduit notamment par le développement systématique des commentaires et des communiqués de presse éclairant ses décisions, par l'initiation de bilans statistiques ${ }^{40}$, par l'enrichissement des tables analytiques relatives à ses décisions ${ }^{41}$, par l'ouverture des archives et, en particulier, l'accès aux procès-verbaux des délibérations, par la retransmission vidéo des plaidoiries exposées dans le cadre des QPC, ou encore par la publication des listes des initiateurs puis des textes des portes étroites ${ }^{42}$. Il s'agit là de l'expression d'une réelle volonté d'accroître la transparence, la diffusion et la vulgarisation, c'est-à-dire l'accessibilité au grand public, des activités de l'institution. Cette évolution a largement été saluée par le milieu académique. Outre qu'elle permet un rapprochement à l'égard de citoyens qui connaissaient mal, voire pas du tout, l'institution et les procédures qui la caractérisent ${ }^{43}$, elle favorise indéniablement la recherche en droit constitutionnel par la mise à disposition d'un ensemble de données jusque-là gardées sous silence ou difficilement accessibles. Il devient par conséquent dorénavant possible de confronter des hypothèses jusqu'alors invérifiables à toute une série d'éléments et de documents fournis par le Conseil constitutionnel ${ }^{44}$.

Parallèlement, le Conseil constitutionnel a également engagé, de manière continue et croissante depuis la fin des années 1980, une activité de collaboration avec le monde scientifique. Elle se traduit de multiples manières: organisation de colloques, publication de revues dédiées à son activité, ou plus largement, au droit constitutionnel (Les cahiers du Conseil constitutionnel, Les nouveaux cahiers du Conseil constitutionnel, puis, aujourd'hui, Titre VII), présence régulière de membres du Conseil constitutionnel dans 
différents cercles universitaires (jurys et prix de thèse, colloques...), parrainage par le Conseil du prix annuel du livre juridique ${ }^{45}$, etc.

\subsection{Questionnements}

24 Ce double positionnement du Conseil constitutionnel - multiplication des éléments de mesure et de connaissance de sa propre activité, d'une part, entretien et développement des échanges avec le monde universitaire, d'autre part - soulève plusieurs questions méthodologiques et épistémologiques, notamment celles, classiques, tenant aux conditions de possibilité d'une analyse universitaire critique, autonome, extérieure à l'institution analysée et aux usages qui peuvent être faits par cette dernière de l'analyse produite. La science politique $a$, de longue date, pensé les enjeux et conditions de l'enquête universitaire sur l'État, le droit et les institutions ${ }^{46}$. Comme le relevait Pierre Bourdieu, «Entreprendre de penser l'État, c'est s'exposer à reprendre à son compte une pensée d'État, à appliquer à l'État des catégories de pensée produites et garanties par l'État, et donc à méconnaître la vérité la plus fondamentale de l'État $»^{47}$.

De ce point de vue, il existe un trait marquant de la doctrine constitutionnelle française qui caractérise aussi mutatis mutandis celle opérant dans le champ du droit administratif ${ }^{48}$. Pour des raisons tenant à la sociologie des élites de gouvernement mais aussi aux représentations dominantes de la fonction de juger et à la difficulté de prendre de la distance avec le mythe du juge bouche de la loi, les analyses doctrinales produites font souvent preuve de déférence, voire de légitimation à l'égard de l'institution et de ses décisions. Beaucoup ont ainsi accompagné et justifié les rôles de régulateur des pouvoirs publics puis de gardien des droits et libertés autour desquels le Conseil constitutionnel a structuré et justifié son activité au moins depuis $1971^{49}$. La doctrine constitutionnaliste française a donc longtemps incarné la difficulté de prendre une distance critique avec son objet d'étude ${ }^{50}$. De ce point de vue l'article de Danièle Lochak paru en 1986, «Le Conseil constitutionnel, protecteur des libertés? $»^{51}$, est précurseur et constitue un article de référence. Alors que beaucoup se rangeaient à l'image de gardien des droits et libertés que le Conseil constitutionnel entendait se donner depuis sa décision de 1971, l'auteure tenait des propos beaucoup plus nuancés : "si le juge constitutionnel s'en tient à la lettre des dispositions constitutionnelles, son contrôle est inefficace et inutile dans la mesure où elles ne fournissent qu'exceptionnellement une réponse à la question posée; si, à l'inverse, il s'efforce de donner vigueur à ces dispositions par une interprétation 'constructive' autrement dit subjective, il s'expose au grief d'arbitraire et à l'accusation de vouloir gouverner. Soucieux d'éviter ces deux écueils, le Conseil constitutionnel pratique la navigation à vue, qui s'accommode mal d'une rigueur juridique au demeurant inaccessible avec les instruments dont il dispose, mais tout aussi peu d'une cohérence politique également illusoire au regard des exigences contradictoires auxquelles il est soumis $»^{52}$. Elle ajoutait plus loin: "Tantôt maniant librement la pétition de principe, tantôt s'enfermant volontairement dans un formalisme juridique étroit, le juge constitutionnel reste maître de sa décision finale. L'incertitude des bases normatives, la fragilité de la démonstration, font inévitablement soupçonner le caractère largement téléologique de sa jurisprudence. Si ce soupçon est fondé, il devient légitime et nécessaire de s'interroger sur les implications concrètes des décisions du Conseil 
constitutionnel, en le suivant sur le terrain qui est véritablement le sien, aux confins du politique et du juridique $»^{53}$.

Ces questionnements se posent dans des termes encore aiguisés dès lors que l'institution à étudier prend elle-même l'initiative et le pilotage d'un appel à projets. Certes, le Conseil constitutionnel s'est d'emblée, pour ce faire, doté d'un conseil scientifique ${ }^{54}$. Celui-ci a été associé tant à la conception qu'à la rédaction de l'appel à projets, puis, à la sélection des projets retenus et à leur suivi pendant les 18 mois de la recherche. Ce procédé n'est pas inusuel au sein de la recherche juridique ${ }^{55}$. Il ne va toutefois pas sans poser quelques interrogations dès lors que, comme on l'a relevé, l'appel à projets à ambition scientifique porte directement sur le fonctionnement et l'activité de l'institution elle-même. Qu'est-ce qui peut en effet le distinguer d'un audit ou d'une expertise? Quel type de distanciation critique peut-il exister entre les équipes de recherche et l'institution, tout particulièrement lorsque l'on connaît les rapports de domination qui prolifèrent dans le champ, conduisant de façon, plus ou moins contrôlée et contenue selon les personnalités, à une révérence du monde académique envers une institution qui le fascine et que côtoyer grandit?

On retrouve ici des difficultés méthodologiques de distanciation analogues à celles mises en avant par les sociologues au sujet des entretiens. Celles et ceux qui concourent à l'appel d'offre sont ipso facto appelés à enquêter et à produire une analyse sur une institution nationale qui, au moins symboliquement, les domine. Or dans quelle mesure est-il possible de s'imposer aux imposants ${ }^{56}$ ? Cette question est d'autant plus aigüe dans la configuration d'un appel d'offre où l'institution étudiée finance et oriente la recherche car, au rapport de domination symbolique, s'ajoute un rapport de demandeur à donneur ou bienfaiteur. Ces difficultés ont très bien été soulignées dans la présentation du projet faite par Guillaume Drago : «L'appréciation que je porte sur ce projet de recherches et ses résultats est positive mais elle doit aussi interroger. Il est clair que ce projet de recherches, porté et financé généreusement par le Conseil constitutionnel s'insère dans une logique institutionnelle et officielle qui a pu conduire à 'lisser' certains résultats, sans pour autant porter atteinte à la liberté intellectuelle et de recherche des équipes ayant porté leurs projets. Ce constat est inévitable mais il n'a aucunement biaisé les résultats. Le système de l'appel à projets comporte des avantages et des inconvénients. (...) Le regret, et c'est un sentiment paradoxal pour un universitaire qui tient plus que tout à préserver la liberté intellectuelle et de recherche, est peut-être de ne pas avoir défini des thèmes plus précis et de solliciter directement des équipes de chercheurs, sur les sujets qui fâchent. (...) Mais on ne peut demander à une institution de pratiquer, par voie de chercheurs interposés, une sorte de 'Séance de la flagellation' intellectuelle $»^{57}$.

\subsection{Hésitations}

A côté de ces questionnements méthodologiques, les hésitations venaient encore d'interrogations relatives aux finalités poursuivies par l'appel à projet et, en dernier ressort, par le Conseil constitutionnel lui-même. L'appel à projet indiquait à cet égard que « les enseignements issus de ces travaux de recherche ont vocation à susciter ou nourrir une réflexion sur l'amélioration du fonctionnement de la QPC, qu'il s'agisse d'éventuelles évolutions des textes encadrant la procédure, ou de réalisations concrètes telles que l'élaboration d'une base de données recensant et analysant les décisions QPC de l'ensemble de la chaîne juridictionnelle (juridictions du fond, Conseil d'État et Cour 
de cassation, Conseil constitutionnel) ». L'appel revendiquait également un objectif de vulgarisation de l'activité du Conseil via la $\mathrm{QPC}^{58}$. «L'accessibilité au plus grand nombre » de certaines des conclusions des recherches était ainsi présentée comme un objectif de l'institution, objectif toujours complexe à cerner, atteindre et réaliser ${ }^{59}$.

29 A dire vrai, c'est de longue date que le Conseil constitutionnel s'appuie sur les insuffisances et critiques relevées par les universitaires pour se réformer et nourrit des échanges avec certains de ses représentants les plus visibles; on peut citer en particulier Louis Favoreu, Dominique Rousseau et Guy Carcassonne. Nombre des innovations et efforts de transparence, de mises à disposition de données, voire d'inflexions jurisprudentielles du Conseil, peuvent être reliés à ce dialogue dans la mesure où ils répondent, plus ou moins directement, à des propositions doctrinales : songeons à l'accès aux procès-verbaux des délibérations ou, plus récemment, aux publications de la liste puis des textes des tierces interventions introduites dans le cadre des recours a priori. Procèdent d'ailleurs d'une même logique d'échange, des demandes qui restent en suspens telles que celle de la publication d'opinions dissidentes.

Ces questionnements et hésitations ont finalement conduit l'UMR 7074 CTAD, qui depuis longtemps se caractérise par des orientations épistémologiques de type analytique et critique, à ne pas prendre part à l'appel à projets émis par le Conseil constitutionnel. Après discussion collective, le choix a été fait de travailler de manière autonome - hors cadre et hors financement par le Conseil - sur le bilan de dix années de QPC. Il en allait aussi, de l'avis de celles et ceux qui se sont impliqués dans le groupe de recherche, d'une certaine liberté temporelle (pas de contrainte d'un livrable à une date imposée) et surtout d'une liberté de traitement du corpus. Espoir a ainsi été d'avoir le champ libre pour tenter de produire un autre regard sur les décisions rendues par le Conseil constitutionnel dans le cadre de la procédure de la QPC depuis dix années.

\section{Approches épistémo-méthodologiques : partis pris et difficultés}

31 L'étude présentée entend proposer une analyse critique (2.1.), empirique et collective (2.2.) de la mise en œuvre de la procédure des QPC. Chacune de ces dimensions a été confrontée à ses difficultés particulières et demandent des précisions.

\subsection{Une approche critique}

32 La démarche engagée a pour visée de produire un ensemble de discours critiques, au sens épistémologique, c'est-à-dire des discours descriptifs et explicatifs qui veillent à se distancier de propos justificatifs de l'institution, de son activité et, plus particulièrement ici, de la procédure des QPC. Cette critique épistémologique entend se départir d'une autre forme de critique, une critique axiologique, qui consisterait à formuler des jugements de valeur sur l'objet étudié. Comme l'ont fort bien expliqué François Ost et Michel van de Kerchove, il est en effet possible de distinguer au moins deux formes de discours critiques sur le droit. La première consiste à formuler des «jugements de valeur dénotant une attitude pratique»: appréciation en termes de bien ou de mal, de bon ou de mauvais, d'acceptable ou de non acceptable, de satisfaisant ou de non satisfaisant, d'efficace ou de non efficace, de juste ou d'injuste. La seconde provient d'un « jugement de réalité dénotant une attitude de connaissance $»^{60}$ : 
appréciation en termes de cohérence ou d'incohérence, de logique ou d'illogique, de consistance des présupposés.... Chacun de ces types de discours critiques produit sa propre forme de rupture et de mode de distanciation à l'égard de l'objet d'étude : d'un côté, une rupture «axiologique " qui conduit à déployer un discours «d'évaluation critique opérée à partir de choix de valeurs alternatifs » et, de l'autre, une attitude de rupture "épistémologique " qui produit des discours d'explication ${ }^{61}$. Ces discours peuvent revêtir différentes formes selon l'objectif de la recherche ou les présupposés épistémologiques, méthodologiques et théoriques retenus. La rupture " avec la réalité telle qu'elle est perçue ou vécue ${ }^{62}$ peut ainsi consister en des opérations plus ou moins liées de "distanciations", de "déplacements", de "dévoilements" ou encore de « changements de point de vue » à l'égard de l'objet observé. Dès lors, il «ne s'agit pas d'apporter une contribution au bon fonctionnement de l'ordre juridique» qui consisterait en l'occurrence à vouloir, dans une perspective normative de la connaissance, améliorer la procédure de QPC et les décisions qu'elle rend possible. L'objectif est plutôt de s'intéresser aux processus de production de ces décisions et, notamment, à les confronter aux objectifs initialement assignés à la procédure de QPC.

Par ailleurs, tout en gardant un statut descriptif et explicatif, l'approche critique proposée se distingue d'opérations de simple réitération technique des énoncés du Conseil constitutionnel. Autrement dit, il ne s'agit pas de répéter, de rationaliser ni de rendre cohérents les décisions étudiées, les données et les discours que le Conseil livre sur ses propres activités. La perspective est de décrire ou d'expliquer autre chose, cette autre chose résultant des choix de corpus et matériaux appelés à être analysés et des types de questionnement qui leur sont adressés. Comme l'expliquent là encore François Ost et Michel van de Kerchove, alors que «la seule description positiviste des règles, notions et institutions juridiques » peut revenir à épouser « fondamentalement le point de vue des acteurs mêmes de la vie juridique, c'est-à-dire le point de vue d'un 'utilisateur' d'un technicien, à la rigueur d'un 'technologue' mais non d'un savant ", «l'objectivité scientifique postule au contraire que l'on change de 'point de vue' et que l'on voie les choses telles qu'elles sont [et non] telle que notre système social nous les laisse voir $»^{63}$.

Dès lors, l'interrogation centrale sur laquelle repose l'approche critique de l'analyse de dix années de QPC est simple : la QPC a-t-elle permis aux justiciables de (mieux) voir protéger les «droits et libertés que la Constitution garantit »? De cette interrogation première découlent de multiples sous-questions connexes qui permettent de guider et de structurer les réflexions d'un programme de recherche. Parmi celles-ci, ont été en particulier retenu :

Quels sont les justiciables s'emparant de cette procédure?:

- Quels sont leurs profils?

- Existe-t-il des disparités entre les recours en QPC en fonction des justiciables (primauté d'un ordre juridictionnel, type de questions soulevées via la QPC, corpus de dispositions contestées, type de représentation des requérants et requérantes, issue des procédures, etc.) ?

Quelles sont les tendances que peut révéler une analyse quantitative systématique quant au rôle joué par les différents acteurs dans la procédure QPC?

- Les avocats s'emparent-ils de la QPC de manière fortuite ou existe-t-il une forme de spécialisation dessinant une captation du contentieux QPC par des cabinets qui y consacrent leur expertise? 
- Comment le Conseil constitutionnel s'approprie-t-il les QPC en redéfinissant le champ des dispositions contestées ou les moyens invoqués?

- Le type de corpus attaqué est-il corrélé avec l'usage de certaines techniques argumentatives auxquelles recourt le Conseil constitutionnel? que la constitution garantit?

- Les décisions QPC ont-elles permis la garantie de droits nouveaux?

- Impliquent-elles un changement dans le niveau de protection des droits ou dans la manière de les protéger?

- Comment le corpus jurisprudentiel QPC contribue-t-il à redéfinir la catégorie de «droits et libertés garantis par la Constitution » via des jeux d'inclusion et d'exclusion catégorielle ou d'intensification et de minoration des niveaux et des formes de protection?

\subsection{Une démarche empirique et collective}

La perspective de dresser un bilan de la procédure QPC supposant de se livrer méthodiquement à une analyse exhaustive du corpus de décisions, il a paru nécessaire d'engager un collectif de chercheuses et de chercheurs sur le projet et, conséquemment, de rendre les données extraites par chacune et chacun exploitables, en s'assurant que l'extraction a été opérée de façon identique.

arche a donc, en premier lieu, exigé de s'assurer que chacun procédait au même travail, c'est-à-dire comprenait, extrayait et rangeait de la même façon les mêmes types de données à partir du lot de décisions qui lui avait été attribué. Ce travail en apparence assez simple à l'échelle individuelle s'est avéré ne pas aller de soi s'agissant d'une équipe d'une dizaine de personnes variablement affûtées au site du Conseil constitutionnel et à la démarche même du codage. La tâche a été de surcroît rendue plus complexe encore en raison de la forme particulièrement indéterminée des concepts sollicités dans l'analyse quantitative qui était d'abord la nôtre. Qu'il s'agisse des techniques argumentatives employées par le Conseil constitutionnel, des concepts qu'il utilise dans ses décisions ou des notions proposées par la doctrine pour en rendre compte $^{64}$, ressortaient en effet régulièrement des divergences d'interprétation entre les différents membres du groupe. Il a donc fallu débattre de ces divergences et trouver une définition commune des techniques et des concepts recouvrant des usages dissemblables et assurer la stabilité de leur identification tout au long du processus de recherche. En bref, un protocole d'objectivation d'interprétations originellement personnelles et subjectives a dû être construit. Une difficulté semblable a également pu surgir en raison des variations sémantiques et conceptuelles du discours objet luimême. Une même expression ne désignant pas systématiquement une même réalité dans les décisions du Conseil constitutionnel, il a fallu s'entendre sur une interprétation commune et surmonter les divergences. La difficulté a encore pu provenir de l'indéfinition de certaines catégories. L'indétermination des propriétés constitutives d'une classe (par exemple « les droits et libertés économiques », « le droit social " ou le "contrôle de proportionnalité ») contraignait à identifier de manière stipulative des critères constitutifs pour stabiliser les interprétations.

La démarche a, en second lieu, conduit à envisager la mobilisation d'un outil méthodologique permettant de synthétiser et de confronter aisément les informations collectées. Cette contrainte a rapidement conduit à s'en remettre à une méthodologie quantitative à même d'appréhender la somme de données collectées, en l'occurrence, à 
l'outil statistique. C'est dans ces circonstances que le projet s'est adjoint l'aide capitale d'un sociologue versé aux méthodologies de codage et d'analyse statistique ${ }^{65}$. Toutefois, soucieux de ce que le recours à ce spécialiste du codage ne reste pas un paravent dissimulant une méthodologie syncrétique approximative, le collectif a entretenu avec lui un travail en va-et-vient constant. Celui-ci a notamment consisté à systématiquement exposer les problèmes et les questions qui se posaient à l'assemblée de juristes pour parvenir à encoder les données de façon pertinente et en tirer des statistiques fiables. Inversement, le chercheur en charge du codage a plusieurs fois exposé ses doutes et difficultés pour ajuster les codages aux modes de catégorisation juridiques et aux besoins de la recherche.

41 Mobiliser conjointement l'analyse juridique, le classement analytique et le codage statistique a contraint les uns et les autres à des efforts considérables d'assimilation et de traduction en vue d'éviter les erreurs d'interprétation et des biais dans les résultats obtenus. Chacun a dû se remettre plusieurs fois à l'ouvrage sur son corpus pour obtenir des données plus fines et des résultats probants. Du côté des juristes, il s'est en particulier agi de sédimenter (c'est-à-dire stabiliser les critères constitutifs) et d'aiguiser (préciser ces critères de sorte à rendre leur application binaire) les catégories en vue de les quantifier. Du côté du codage, il s'est agi de coder (ou quantifier) l'ensemble des données fournies sans perdre de vue leur imbrication réciproque et les réalités juridiques qu'ils recouvrent. Des erreurs et imprécisions qui ont un temps pu avoir cours dans la discussion ont ainsi donné lieu à des anomalies plus ou moins cocasses pour qui a une vision générale préalable du contentieux constitutionnel, comme le fait par exemple de retrouver certaines années $80 \%$ de décisions modulant leur application dans le temps ou des années où $10 \%$ seulement des requérants étaient représentés par un cabinet, etc. Il s'est vite avéré que ces anomalies résultaient d'erreurs de traduction. Elles ont alors fait l'objet de corrections permises par de nouveaux échanges et d'une reprise du travail de codage. A cet égard, il a été éprouvé de manière d'autant plus forte que le corpus d'analyse était vaste (730 décisions) le fait que le travail en commun entre spécialistes de disciplines différentes nécessite la construction d'une passerelle solide de dialogue. Celle-ci ne s'établit, ni ne se règle une fois pour toute en amont de la recherche. Elle nécessite des moments réguliers, mais non entièrement programmables à l'avance, d'ajustement et de réajustement. Ce processus d'auto-correction au long cours ne trouve probablement d'ailleurs jamais de fin. S'il faut bien se résoudre à exploiter à un moment donné les résultats collectés, il apparaît toujours possible, sinon probable, que des erreurs persistent lesquelles suggèreront le cas échéant de nécessaires améliorations du corpus statistique.

En dépit de ce caractère irrémédiablement dynamique, la chronologie d'exploitation du matériau d'analyse et de production de données peut, en l'occurrence, être schématiquement ainsi retracée :

\section{Matériau brut-> Donnée juridique brute extraite de l'analyse -> Codage statistique -> Donnée statistique résultant du croisement des codages-> Décodage en donnée juridique exploitable}

Ce mouvement suggère que la donnée, après avoir été encodée statistiquement, a de nouveau été « traduite » pour servir l'analyse juridique. Cette dernière étape ne va pas non plus sans difficulté pour le juriste qui se trouve confronté, si ce n'est contre son 
gré, du moins contre son habitude, à des dizaines de pages de tableaux statistiques. Le dépaysement peut rapidement laisser place à la confusion. La proposition de multiples tris croisés opérés par le statisticien entre les différentes données établies (par exemple corrélation entre type de requérant et modulation des effets dans le temps, entre cabinet d'avocat et techniques argumentatives utilisées par le Conseil constitutionnel) n'a ainsi pas été sans provoquer un certain désemparement. Un travail d'échanges et d'interprétation collectifs des résultats statistiques s'est donc encore avéré nécessaire pour identifier des anomalies, uniformiser les interprétations et dégager des lignes de force intéressantes pour les juristes et l'analyse plus particulière de dix années de Question prioritaire de constitutionnalité.

Si donc, le présent travail résulte évidemment d'efforts individuels, il transcrit également les discussions et les débats parfois vifs qui ont animé le collectif au gré de l'avancement du projet. La démarche méthodologique adoptée, inédite pour la plupart d'entre les chercheurs impliqués, était résolument expérimentale et constructive. L'idée générale a été de faire remonter les interrogations et les difficultés rencontrées individuellement au groupe afin d'en débattre de manière collégiale et de trouver, le cas échéant, une issue satisfaisante compatible avec la finalité du projet.

\section{NOTES}

1. Présentée comme « une véritable révolution juridique et un droit nouveau » par le Président de la République (Discours prononcé au Conseil constitutionnel le $1^{\mathrm{er}}$ mars 2010), ou comme "la principale avancée des droits et des libertés dans notre pays depuis l'abolition de la peine de mort » selon les mots du Président du conseil constitutionnel (Allocution de Jean-Louis Debré au Conseil National des Barreaux du 21 octobre 2011).

2. 2010-1 QPC, 28 mai 2010.

3. Les chiffres de la QPC diminueront par la suite, 2010 et 2011 étant analysées comme des années de fort engouement vis-à-vis d'une réforme qui avait été attendue et préparée. Depuis 2012, la moyenne annuelle de décisions QPC se stabilise aux alentours de 65.

4. Pascal Jan, "Comprendre la vie publique à travers la Constitution ", sur le site vie-publique.fr, 9 mars 2016 : https://www.vie-publique.fr/parole-dexpert/268599-comprendre-la-vie-publiquetravers-la-constitution-du-4-octobre-1958

5. V. notamment la décision par laquelle la Cour décide de renvoyer la QPC devant la CJUE pour que soit vérifiée sa compatibilité au droit de l'Union européenne (Cass, QPC, 16 avril 2010, $\mathrm{n}^{\circ}$ 10-40.002, Melli et Abdeli) ou encore la décision par laquelle elle refuse de transmettre une QPC relative à la loi Gayssot (Cass., QPC, 7 mai 2010, n 09-80.774, Rivarol) ou précise qu'une QPC ne peut pas porter sur l'interprétation d'une disposition législative (Cass., crim., 19 mai 2010, $\mathrm{n}^{\circ}$ 09-82582).

6. V. le colloque qui s'est tenu à l'Assemblée nationale le 05 avril 2013 : «La QPC, une question pour la démocratie ».

7. Christine Maügué, «La QPC : 5 ans déjà et toujours aucune prescription en vue ", Nouveaux cahiers du Conseil constitutionnel, $\mathrm{n}^{\circ} 47$ (Dossier : 5 ans de QPC), avril 2015, pp. 11-26; V. Aussi : Julien Bonnet, Pierre-Yves Gahdoun dir., La QPC : une révolution inachevée ?, LGDJ Varenne, 2016. 
8. Julien Bonnet, Pierre-Yves Gahdoun, La question prioritaire de constitutionnalité, PUF, Coll. Que sais-je ?, 2014.

9. Dominique Rousseau, Droit du contentieux constitutionnel, $10^{\text {ème }}$ ed., Montchrestien, 2013, p. 44.

10. V. https://www.conseil-constitutionnel.fr/publications/titre-vii/qpc-2020-les-statistiquesdu-conseil-constitutionnel

11. Mme Nicole Maestracci a présidé le Conseil scientifique du projet QPC 2020, dans le cadre duquel 16 groupes de recherche universitaires ont travaillé. Leurs résultats ont été présentés au cours d'un séminaire fermé qui s'est tenu au Conseil constitutionnel le 26 février 2020, et devaient nourrir l'essentiel d'une manifestation publique d'ampleur repoussée en raison de la pandémie de Covid-19. Les contributions sont disponibles en ligne sur le site du Conseil constitutionnel (lien), ainsi que, sous forme résumée, dans un numéro hors-série de la revue Titre VII.

12. Ce corpus n'intègre pas les "quasi-QPC » dont les critères de recevabilité sont appréciés directement par le Conseil constitutionnel lui-même dans le cadre de son office de juge électoral, sans filtrage des cours suprêmes (voir par exemple la décision n 2014-4909 SEN du 23 janvier 2015, Yonne).

13. Données au 31 décembre 2019 ; v. : https://www.conseil-constitutionnel.fr/bilan-statistique 14. On peut ajouter à ce sous-total 6 décisions rendues relatives à la constitutionnalité de lois du pays, non comptabilisées ici.

15. CC, 3 déc. 2009, $\mathrm{n}^{\circ}$ 2009-595DC, cons. 17 ; et voir ensuite, dès sa première décision QPC : « afin de préserver l'effet utile de la présente décision à la solution des instances actuellement en cours... », CC, 28 mai 2010, n² 2010-1 QPC.

16. «[...] en principe, la déclaration d'inconstitutionnalité doit bénéficier à l'auteur de la question prioritaire de constitutionnalité et la disposition déclarée contraire à la Constitution ne peut être appliquée dans les instances en cours à la date de la publication de la décision du Conseil constitutionnel »: CC., 25 mars 2011, n 2010-108 QPC ; CC, 25 mars 2011, n² 2010-110 QPC. 17. Stéphane Mouton, Mathieu Carpentier dir., L'effet utile des décisions QPC, Rapport de recherche remis au Conseil constitutionnel dans le cadre du projet QPC 2020, 2020, p. 8.

18. Véronique Champeil-Desplats, «L'état d'urgence devant le Conseil constitutionnel, ou quand l'Etat de droit s'accommode de normes inconstitutionnelles ", in Stéphanie Hennette Vauchez dir., Ce qui reste(ra) toujours de l'urgence, LGDJ/Varenne, 2018, p. 151 ; v. Aussi Olga Mamoudy, "L'effet inutile des décisions QPC dans le cadre de la dernière application de l'état d'urgence », in S. Mouton, M. Carpentier dir., L'effet utile des décisions QPC, op. cit., p. 161.

19. Dont on trouve notamment une analyse détaillée in : Véronique Champeil-Desplats, «L'état d'urgence devant le Conseil constitutionnel, ou quand l'Etat de droit s'accommode de normes inconstitutionnelles », op. cit.

20. Christine Maugüé, « La QPC : 5 ans déjà et toujours aucune prescription en vue », op. cit..

21. Les données statistiques publiées sur le site du Conseil constitutionnel diffèrent légèrement, et indiquent 388 saisines QPC émanant du Conseil d'Etat, et 451 émanant de la Cour de cassation. Le différentiel s'explique par le fait que le Conseil constitutionnel comptabilise l'ensemble des saisines, y compris lorsqu'elles sont jointes pour ne faire l'objet que d'une décision.

22. Le delta de 1 s'explique par le fait que, dans les 730 décisions QPC formant le corpus d'analyse, l'une est une décision rectificative qui n'a, dès lors, pas été renvoyée en tant que telle par l'une des juridictions suprêmes.

23. Pascale Deumier, «Autopsie d'une polémique : la QPC, la Cour de cassation et la doctrine », in Mélanges en l'honneur de Jean Louis Bergel, Bruylant, 2013, p. 171.

24. Art. 61-1 de la Constitution.

25. Olivier Duhamel, « La QPC et les citoyens » in Pouvoirs, $n^{\circ}$ 137, avril 2011, p. 188.

26. V. par exemple : Xavier Dupré de Boulois, «Les droits fondamentaux des personnes morales $1^{\text {ère }}$ partie ", RDLF, 2011, chron. $\mathrm{n}^{\circ} 15$ et « Les droits fondamentaux des personnes morales $-2^{\text {nde }}$ 
partie ", RDLF, 2011, chron. $\mathrm{n}^{\circ} 17$; Bertrand Faure, «Les droits fondamentaux des personnes morales ", RDP, 2008, $\mathrm{n}^{\circ} 1$, p. 233.

27. On songe ainsi par exemple à nombre des QPC « état d'urgence » portées notamment par la SCP Spinosi et Sureau (qui assure également la représentation des intérêts de la Ligue des droits de l'Homme), qui soulevaient la question de l'atteinte aux droits fondamentaux causée par la loi du 3 avril 1955 relative à l'état d'urgence.

28. Pour mémoire, la décision de non-renvoi d'une QPC est insusceptible de recours.

29. Cf. Le titre d'un article rédigé par l'un de ces avocats faisant office de prophétie autoréalisatrice dans le contentieux de la QPC, Emmanuel Piwnica, «L'appropriation de la QPC par ses acteurs ", Pouvoirs, 2011, n 137, p. 169.

30. Christine Maugüé, «La QPC : 5 ans déjà et toujours aucune prescription en vue », op. cit..

31. Finalement, les catégories avec lesquelles nous avons travaillé sont les suivantes: droit fiscal, droit de la procédure pénale, droit des entreprises, droit administratif général, droit du travail, droit pénal, droit des collectivités territoriales, droit pénal des affaires, droit des étrangers, droit de la communication, droit de l'outremer, droit pénitentiaire, autres.

32. V. le rapport : Anne-Laure Cassard-Valembois, Régis Vabres dir., QPC et droit fiscal. Les apports croisés du droit fiscal et de la QPC, janv. 2021, https://www.conseil-constitutionnel.fr/sites/default/ files/2020-10/202010_qpc2020_synthese_bourgogne.pdf

33. V. par exemple: Xavier Dupré de Boulois, «La QPC comme supermarché des droits fondamentaux ou les dérives du contentieux objectif des droits", RDLF, 2014, chron. $\mathrm{n}^{\circ} 2$; Stéphanie Hennette Vauchez, «'... les droits et libertés que la Constitution garantit' : quiproquo sur la QPC? », La Revue des droits de l'Homme, 2016, n 10 ; Arnaud Sée, «La QPC et les libertés économiques ", JCP G, 2014, $\mathrm{n}^{\circ} 7$ 18, étude 5.

34. Précisons que nous n'avons codé comme révélant l'exercice du triple test du contrôle de proportionnalité que celles des décisions où le Conseil recourt explicitement à la notion de proportion d'une part, et aux indicateurs de la nécessité, de l'adaptation et de la proportionnalité d'autre part.

35. CC, 27 juin 2001, $\mathrm{n}^{\circ}$ 2001-446 DC.

36. Art. 62 de la Constitution: «Une disposition déclarée inconstitutionnelle sur le fondement de l'article 61-1 est abrogée à compter de la publication de la décision du Conseil constitutionnel ou d'une date ultérieure fixée par cette décision. Le Conseil constitutionnel détermine les conditions et les limites dans lesquelles les effets que la disposition a produits sont susceptibles d'être remis en cause ".

37. Ce que, dès sa première décision $\mathrm{QPC}$, le Conseil constitutionnel nomme « l'effet utile » de ses décisions : v. décision $n^{\circ}$ 2010-1 QPC du 28 mai 2010, Consorts L. (cons. 12)

38. « Appel à projet du Conseil Constitutionnel : 2010-2020: 10 ans de QPC », p. 1.

39. «[...] le Conseil constitutionnel souhaite susciter et soutenir une série de travaux de recherche qui permette de dresser un bilan d'étape, fondé sur des données scientifiques rigoureuses tout en étant accessible à un public large. Ces travaux auront notamment pour objectif d'éclairer des aspects encore insuffisamment documentés de la QPC », Ibid., p. 1.

40. Visant à «donner une vue synthétique de l'activité juridictionnelle du Conseil constitutionnel », https://www.conseil-constitutionnel.fr/les-decisions/comprendre-lesstatistiques.

41. https://www.conseil-constitutionnel.fr/actualites/tables-analytiques-des-decisions-duconseil-constitutionnel-actualisees-au-30-juin-2020.

42. Communiqué de presse du 24 mai 2019 sur les contributions extérieures, https://www.conseilconstitutionnel.fr/actualites/communique/le-conseil-constitutionnel-rendra-desormaispubliques-les-contributions-exterieures-qu-il-recoit.

43. En témoigne l'enquête BVA menée sur le rapport des Français à la QPC. Cette méconnaissance, de l'aveu même du Président du Conseil Constitutionnel, Laurent Fabius, 
« [montre] bien à quel point le succès de la question citoyenne sur les dix dernières années reste à nuancer » autant qu'elle met en exergue le besoin pour l'institution d'accroître « une notoriété qui reste à construire» («Communiqué de presse du 12 novembre 2020 : Enquête grand public BVA - 10 ans de la QPC», https://www.conseil-constitutionnel.fr/actualites/communique/ communique-de-presse-du-12-novembre-2020-enquete-grand-public-bva-10-ans-de-la-qpc).

44. V. par exemple, Anna Marie Lecis Cocco Ortu, Les interventions des tiers porteurs d'intérêts collectifs dans les contentieux constitutionnels incidents français et italien, LGDJ, 2018.

45. Ce prix (https://www.conseil-constitutionnel.fr/evenements/prix-du-livre-salon-du-livrejuridique) est décerné annuellement lors du Salon du livre juridique, lui-même organisé conjointement par le Conseil constitutionnel et le Club des juristes, qui se présente comme un think tank juridique réunissant universitaires et praticiens du droit ( https:// www.leclubdesjuristes.com/ ).

46. Bastien François, « chapitre » in Jacques Commaille, Laurence Dumoulin, Cécile Robert (dir.), La juridicisation du politique, LGDJ, 2010, p. XXX.

47. Pierre Bourdieu, «Esprits d'État. Genèse et structure du champ bureaucratique ", Actes de la recherche en sciences sociales, 1993, $n^{\circ}$ 96-97, p. 49.

48. Cette coïncidence s'explique par le partage d'une conception plus générale du rôle de la doctrine comme « prologue silencieux à toute décision de justice » (Ronald Dworkin, Law's Empire, éd. Hart's Publishing, 1998, p.90, notre traduction). Une telle conception se caractérise par l'identification d'un rapport de complémentarité fort entre le juge et la doctrine (v. notamment, Jacques Chevallier, "Les interprètes du droit ", in La doctrine, éd. CURAPP, 1993, p. 269-270), le premier offrant à la seconde par le truchement de la jurisprudence un matériau essentiel à ses constructions techniques expertes (v. Jacques Caillosse, "Sur quelques problèmes actuels du droit administratif français ", AJDA, 2010, p. 935-936), tandis que celles-ci inspireraient en retour le juge administratif en lui « montrant la voie » (Bernard Pacteau, « La doctrine, auteurs, acteurs $\mathrm{du}$ droit administratif ", in Les Facultés de Droit inspiratrices du droit? [en ligne], Presses de l'Université Toulouse 1 Capitole, 2005, §92-106). La déférence de la doctrine se justifie dès lors autant par sa volonté d'influer sur le cours de la jurisprudence dont elle se saisit que par le besoin impérieux de ne pas rompre le lien avec l'assise empirique sur laquelle elle bâtit son propre édifice théorique.

49. On lira ici avec profit l'important chapitre «Understanding French Constitutional Politics », in Alec Stone Sweet, The Birth of Judicial Politics in France, Oxford University Press, 1992, pp. 96-116. L'auteur y analyse le rôle de la doctrine juridique dans la légitimation du Conseil constitutionnel.

50. V. Bastien François, «Le Conseil constitutionnel et la Vème République ", Revue Française de Science Politique, 1997, vol. 47, n 3, p. 388.

51. Danièle Lochak, « Le Conseil constitutionnel, protecteur des libertés? », Pouvoirs, $\mathrm{n}^{\circ} 13,1986$, p. 41 ; voir aussi, Danièle Lochak, «Les trompe-l'oeil du Palais-Royal », Plein Droit, octobre 1993 mars 1994, n²2-23, p. 6 ; Véronique Champeil-Desplats, « Le Conseil constitutionnel, protecteur des droits et libertés? ", Cahiers de la recherche sur les droits fondamentaux [Online], 9| 2011, http:// journals.openedition.org/crdf/5380.

52. Ibid., p. 42.

53. Ibid., p. 47.

54. Composition du Conseil scientifique: Nicole Maestracci (présidente) et, pour le Conseil constitutionnel : Dominique Lottin (membre), Michel Pinault (membre), Jean Maïa (secrétaire général) ; pour les juridictions: Mattias Guyomar (Conseil d'Etat), Nicolas Bonnal et Nicolas Maziau (Cour de cassation); pour l'Université : Guillaume Drago (Paris II), Stéphanie Hennette Vauchez (Nanterre), Philip Milburn (Lille), Nicolas Molfessis. Etaient également membres Katia Martin Chenut, chercheure au CNRS et directrice-adjointe de la mission de recherche Droit \& Justice, et Christian Behrendt, membre du Conseil d'Etat belge et professeur associé l'Université catholique de Louvain. Naturellement, la participation de Stéphanie Hennette-Vauchez au conseil 
scientifique se serait interrompue si l'UMR CTAD de l'Université Paris Nanterre avait candidaté à l'appel à projets.

55. La mission de recherche Droit \& Justice, par exemple, est une émanation du Ministère de la justice qui semblablement publie de nombreux appels à projets et finance annuellement de nombreuses recherches universitaires.

56. Stéphane Beaud, Florence Weber, Guide de l'enquête de terrain, Paris, La Découverte, 1997, p. 280 ; Hélène Chamboredon, Fabienne Pavis, Muriel Surdez, Laurent Willemez, «S'imposer aux imposants. A propos de quelques obstacles rencontrés par des sociologues débutants dans la pratique et l'usage de l'entretien ", Genèses, 1994, n 16, p. 125 ; S. Cohen, dir., "L'interview démystifiée ", L'art d'interviewer les dirigeants, PUF, 1999, p. 4 et s. Pour une application au Conseil constitutionnel, voir Michael Koskas, Le Conseil constitutionnel par lui-même. Une approche pragmatique des sources du droit, Thèse (en cours), Paris Nanterre, soutenance prévue en décembre 2021.

57. Guillaume Drago, «QPC 2020 : une démarche scientifique pour une vaste ambition », Horssérie, Titre VII, p. 26.

58. «[...] Si la rigueur scientifique constitue la première des qualités attendues, l'accessibilité au plus grand nombre est également souhaitée, notamment en vue de la diffusion des principaux enseignements de ces travaux à un public dépassant la seule communauté juridique ", "Appel à projet du Conseil Constitutionnel : 2010-2020:10 ans de QPC », p. 4.

59. V. notamment, Bernard Ancori, «Vulgarisation scientifique, increasing knowledge gap et épistémologies de la communication ", in Nouvelles perspectives en sciences sociales, 11 (2), 2016, pp. 181-232. https://doi.org/10.7202/1037107ar

60. François Ost, Michel van de Kerchove, Jalons pour une théorie critique du droit, Bruxelles, Publications des Facultés universitaires Saint-Louis, 1987, p. 27.

61. François Ost, Michel van de Kerchove, ibidem, p. 29.

62. Jacques Chevallier, Danièle Lochak, Science administrative, Paris, LGDJ, 1978, t. 1, p. 83.

63. François Ost, Michel van de Kerchove, op. cit., p. 55.

64. Voir, de manière emblématique, la notion d'effet cliquet «artichaut ». Pour un exemple de son usage: Julien Dellaux, «La validation du principe de non-régression en matière environnementale par le Conseil constitutionnel au prix d'une redéfinition a minima de sa portée ", Revue juridique de l'environnement, 2017/4, pp. 693-704; et pour une critique : Dominique Chagnollaud, «Au Panthéon des concepts 'fourre-tout': le syndrome de l'artichaut », Droits, 2006/2, n 44, pp. 93-100.

65. L'équipe de recherche remercie très vivement $M$. Jérôme Pacouret pour son aide inestimable pour le codage, la constitution d'une base générale, et la réalisation de multiples tris à plat et tris croisés.

\section{RÉSUMÉS}

Ce texte introductif présente les questions de recherche sur la base desquelles le projet de bilan de 10 années de QPC a été mené au sein du CREDOF ; en particulier, il s'est agi de confronter une analyse exhaustive du corpus de 730 décisions rendues par le Conseil constitutionnel depuis 2010 aux finalités et objectifs qui avaient été assignés à cette nouvelle voie de droit par ses 
promoteurs. La méthodologie de la recherche est elle aussi explicité : constitution du corpus, catégories de classement et d'analyse, travail collectif, traitement statistique.

This introductory text presents the main research questions that have grounded the project of taking stock of 10 years of QPC litigation within the CREDOF. In particular, we have sought to confront an exhaustive analysis of all the 730 decisions delivered by the Conseil constitutionnel since 2010 within this new legal procedure to the finalities and objectives it was assigned by its promoters. The methodology is also explicited here : how the rulings were assembled, how they were classified and analyzed, statistical analyses, throughout a collective and collaborative research endeavour.

\section{INDEX}

Mots-clés : QPC, droit et libertés, méthode quantitative, contrôle de constitutionnalité, Conseil constitutionnel, statistiques

Keywords : QPC, rights and freedoms, quantitative method, constitutional review, Constitutional council, statistics 